\title{
Non-destructive extraction of DNA from preserved tissues in medical collections
}

Rayo, Enrique ; Ferrari, Giada ; Neukamm, Judith ; Akgül, Gülfirde ; Breidenstein, Abagail M ; Cooke, Martyn ; Phillips, Carina ; Bouwman, Abigail S ; Rühli, Frank J ; Schuenemann, Verena J

\begin{abstract}
Museum specimens and histologically fixed material are valuable samples for the study of historical soft tissues and represent a possible pathogen-specific source for retrospective molecular investigations. However, current methods for molecular analysis are inherently destructive, posing a dilemma between performing a study with the available technology, thus damaging the sample, and conserving the material for future investigations. Here the authors present the first tests of a non-destructive alternative that facilitates genetic analysis of fixed wet tissues while avoiding tissue damage. The authors extracted DNA from the fixed tissues as well as their embedding fixative solution, to quantify the DNA that was transferred to the liquid component. The results show that human historical DNA can be retrieved from the fixative material of medical specimens and provide new options for sampling valuable collections.
\end{abstract}

DOI: https://doi.org/10.2144/btn-2021-0014

Posted at the Zurich Open Repository and Archive, University of Zurich

ZORA URL: https://doi.org/10.5167/uzh-214298

Journal Article

Published Version

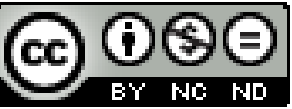

The following work is licensed under a Creative Commons: Attribution-NonCommercial-NoDerivatives 4.0 International (CC BY-NC-ND 4.0) License.

Originally published at:

Rayo, Enrique; Ferrari, Giada; Neukamm, Judith; Akgül, Gülfirde; Breidenstein, Abagail M; Cooke, Martyn; Phillips, Carina; Bouwman, Abigail S; Rühli, Frank J; Schuenemann, Verena J (2022). Non-destructive extraction of DNA from preserved tissues in medical collections. BioTechniques, 72(2):60-64.

DOI: https://doi.org/10.2144/btn-2021-0014 


\section{Non-destructive extraction of DNA from preserved tissues in medical collections}

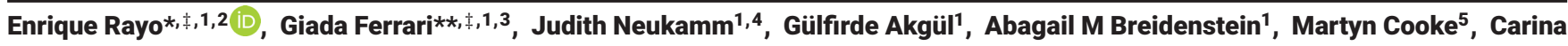
Phillips $^{5}$, Abigail S Bouwman' ${ }^{1}$, Frank J Rühli \& Verena J Schuenemann ${ }^{1}$

${ }^{1}$ Institute of Evolutionary Medicine (IEM), University of Zürich, Zürich, CH-8050, Switzerland; ${ }^{2}$ Plant Ecology Genomics Group, Institute of Integrative Biology (IBZ), ETH Zürich, CH-8092, Switzerland; ${ }^{3}$ Royal Botanical Garden Edinburgh, Edinburgh, EH3 5NZ, UK; ${ }^{4}$ Institute for Archaeological Sciences, University of Tübingen, Tübingen, DE-72074, Germany; ${ }^{5}$ Museums \& Archives, The Royal College of Surgeons of England, London, WC2A 3PE, UK; *Author for correspondence: enrique.rayo@iem.uzh.ch; ${ }^{\star *}$ Author for correspondence: gferrari@rbge.org.uk; ${ }^{\ddagger}$ Both authors contributed equally

BioTechniques 72: 00-00 (February 2022) 10.2144/btn-2021-0014

First draft submitted: 19 February 2021; Accepted for publication: 16 December 2021; Published online: 17 January 2022

\section{ABSTRACT}

Museum specimens and histologically fixed material are valuable samples for the study of historical soft tissues and represent a possible pathogen-specific source for retrospective molecular investigations. However, current methods for molecular analysis are inherently destructive, posing a dilemma between performing a study with the available technology, thus damaging the sample, and conserving the material for future investigations. Here the authors present the first tests of a non-destructive alternative that facilitates genetic analysis of fixed wet tissues while avoiding tissue damage. The authors extracted DNA from the fixed tissues as well as their embedding fixative solution, to quantify the DNA that was transferred to the liquid component. The results show that human historical DNA can be retrieved from the fixative material of medical specimens and provide new options for sampling valuable collections.

\section{METHOD SUMMARY}

The authors compared the metagenomic content of historical tissues and their embedding liquid to retrieve DNA from the host and specified pathogens based on the diagnosis of the sample. They applied ancient DNA research techniques, including in-solution hybridization capture with DNA baits for human mitochondrial DNA, Mycobacterium tuberculosis, Mycobacterium leprae and Treponema pallidum.

\section{KEYWORDS:}

ancient DNA • historical DNA • metagenomics • museomics

Fixed wet tissues from museums and anatomical collections offer an extensive, often pathogen-specific and precisely dated archive for retrospective molecular investigations. Although most collections are accessible for collaborative, scientific inquiry, destructive methods such as sampling for molecular analysis should always be carefully reviewed. For this reason, developing non-invasive sampling methods for specimens in museums or medical collections has been a central objective of the paleogenetics field for decades [1-3], but even in those cases a minimal intrusion and retrieval of organic material is necessary. Traditional extraction techniques on preserved tissues [4] rely on the binding of DNA strands to inorganic structures, such as bone or teeth, or the presence of enough preserved genetic material within soft tissues. The retrieval of DNA is then achieved by homogenizing and chemically dissolving the tissue. However, DNA from organisms has been reported to survive freely in an array of different ecosystems, such as soil, freshwater, or saltwater [5,6]. This raises the question of whether the same leaching principle is observable with specimens preserved in embedding liquid for a long period of time, such as for medical and archival collections, where tissues of interest are preserved in the same ethanol or formalin preservative solution for decades or even centuries. If DNA from long-time preserved specimens, usually referred to as historic and ancient DNA (aDNA), leached into the fixative liquid, it would represent a less invasive substrate to sample to obtain human and pathogen aDNA for molecular analyses than the tissue itself. The molecular detection of leached DNA from a preserved specimen has been tested previously, but only with traditional PCR methods and very recent material - 7-10 years old [7]. To the authors' knowledge, no other study has assessed the feasibility of characterizing aDNA utilizing the embedding liquid of historical collections.

Here the authors present results from an exploratory study on fixed specimens from the museum collections at the Royal College of Surgeons (RCS) in London (UK). These collections contain approximately 3500 18th century preparations from the original collection of surgeon and anatomist John Hunter and 7500 other preserved tissue preparations ranging from the 19th century to the present day. The authors collected tissue and fixative samples from ten specimens (14 in total with some replicates subsampled) dated between 1760 and $1886 \mathrm{CE}$. The selected individuals were diagnosed with tuberculosis, leprosy, syphilis, cancer and skin conditions (Supplementary Table 1). These historical samples represent good candidates for a retrospective genomic analysis of these disease-causing pathogens: namely, members of the Mycobacterium tuberculosis complex (MTBC), Mycobacterium leprae and Treponema pallidum subsp. pallidum. With metagenomic shotgun sequencing data obtained from tissues as a baseline, the authors assessed the retrieval of human and 


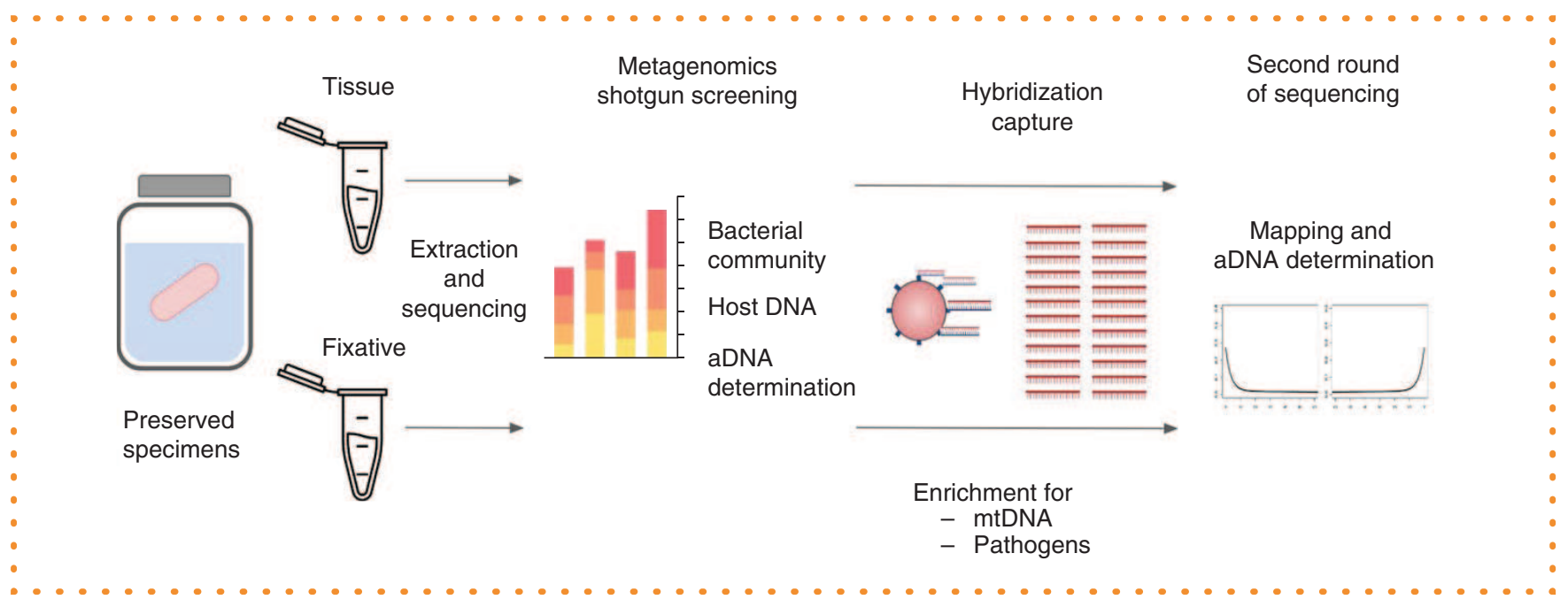

Figure 1. Schematic representation of the workflow of the project.

candidate pathogen DNA from the corresponding fixatives, using both a shotgun sequencing approach and hybridization capture of the human mitochondrial (mtDNA) and bacterial pathogen DNA (overview in Figure 1) (Supplementary Notes 1 \& 2).

For the first assessment of ancient DNA preservation in the samples, the authors screened the shotgun sequencing output for reads that could be mapped against the human mtDNA. Nine out of 14 tissue replicates showed detectable traces of mtDNA, eight with a $5 \mathrm{X}$ coverage above $98 \%$ and one above $24 \%$ (Supplementary Table 5). These replicates demonstrate the characteristic misincorporation patterns for ancient DNA (elevated $\mathrm{C}$ to $\mathrm{T}$ substitutions at $5^{\prime}$-ends and elevated $\mathrm{G}$ to $\mathrm{A}$ substitutions at $3^{\prime}$-ends) in frequencies ranging from $10 \%$ to $20 \%$ damage [8]. Conversely, only the liquid sample HA4.1 showed DNA that could be considered as ancient $(94.59 \% 5 \times$ coverage of mitogenome, 16\% damage). The enrichment strategy [9] (SI Note 2) was robust enough to increase the endogenous content of all the tissue samples (Supplementary Table 6), with fold increments from 14 to 275, and for eight of the ten liquid samples (Supplementary Table 7), not including HA4.1. This sample had a remarkable increase with a final output of more than three million mapped ancient mtDNA reads, a 537 -fold increase in the endogenous content (100\% coverage), and retaining damage of $10 \%$ (Figure $2 \mathrm{~A}$ ).

Only seven tissue samples and one liquid sample (HA4.1) had sufficient reads and coverage for mitochondrial haplotype assignment and contamination estimation (Supplementary Table 8). The HA4.1 tissue sample was characterized as the Eastern European haplogroup $\mathrm{H} 2 \mathrm{a} 2$, and the corresponding liquid sample was assigned to the same haplogroup but to a higher definition due to the high number of reads retrieved ( $\mathrm{H} 2 \mathrm{a} 2$ subgroup a1), confirming the transfer of mtDNA from the tissue to the embedding fixative material. An estimated $0.01 \%$ of reads from HA4.1 were identified as contaminated by haplogroup K1 [10]. This could be due to handling, either during tissue preparation or further curation treatments (e.g., refilling the glass containers), but it is still a very low percentage of contaminant reads. For the rest of the tissue samples, the haplogroups identified were also typically European, which was not a surprise, since the samples were expected to be of European origin (Supplementary Table 8) [11,12].

Bacterial detection for shotgun screening, on the other hand, was less satisfactory. The metagenomic analysis showed certain sample-dependent variations in the number of mapped bacterial reads, with a general higher abundance of bacterial reads in the tissue (from 300,000 to 1,200,000) compared with the ethanol (ranging from 50,000 to 650,000) (Figure 2B). The authors estimated richness by calculating the bacterial alpha diversity using the observed and Shannon indexes [13]. Tissue samples consistently scored higher for both indexes (Supplementary Figure 1).

At the phylum level, the tissue samples are heavily dominated by Proteobacteria (from 55 to $99 \%$ of total reads). Liquid samples are also dominated by Proteobacteria (20-78\%), but with Firmicutes (53\% in some samples) and Actinobacteria (12-32\%) in higher relative abundance (Figure 2C). At the family level, some tissue samples were heavily dominated by members of Enterobacteriaceae (75-95\%), while others were higher for Pseudomonadaceae (20\%), Comamonadaceae (7-20\%) and Burkholderiaceae (5-15\%). The fixative samples had a more homogeneous abundance distribution of the main families, with Burkholderiaceae (10-27\%), Clostridiaceae (5-55\%) and Micromonosporaceae (5-28\%) present among all samples. A noticeable presence of Clostridium in the liquid samples was detected, higher in the ethanol-based fixative (ranging from 4-56\%) but very low in the Kaiserling fixative HA4.1E ( $<1 \%)$. Clostridium was absent from or barely detectable in the tissue samples. At the species level, there were not enough reads to be reliably mapped to the reference genome, and no taxa presented a damage pattern to be validated as ancient. The families mentioned above are widespread in most environments [14-16], and members of Clostridium are nearly ubiquitous in nature and very resistant to heat, desiccation and toxic chemicals [17]. This suggests that most of the bacteria in these samples were present due to environmental contamination. The only sample that showed a shared component of bacteria with its respective tissue sample was HA4.1, with a significant abundance 


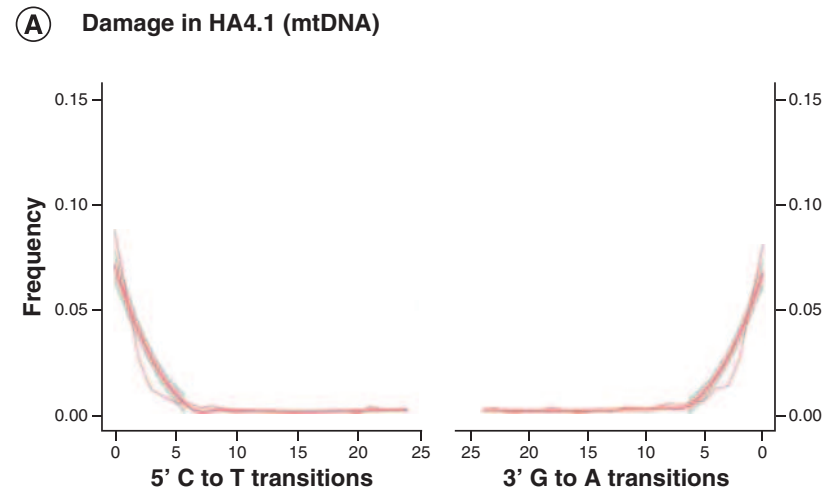

(C) Phyla abundance expressed in percentage

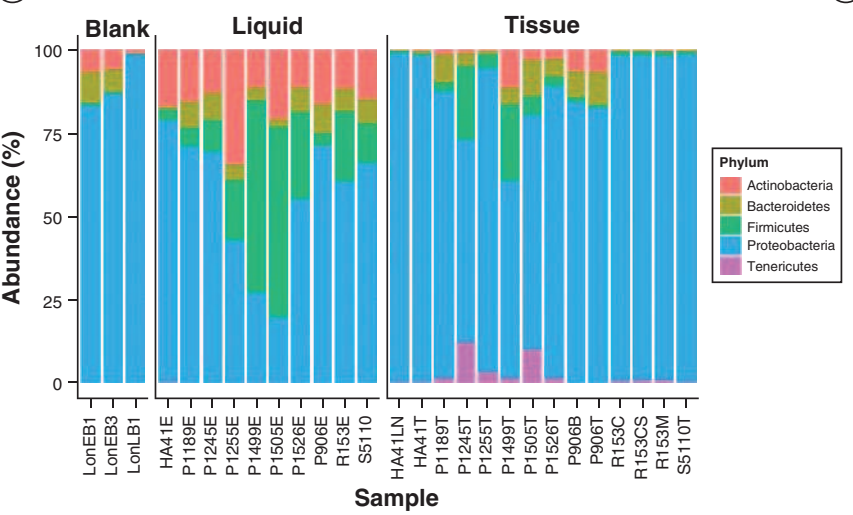

(B) Total number of reads

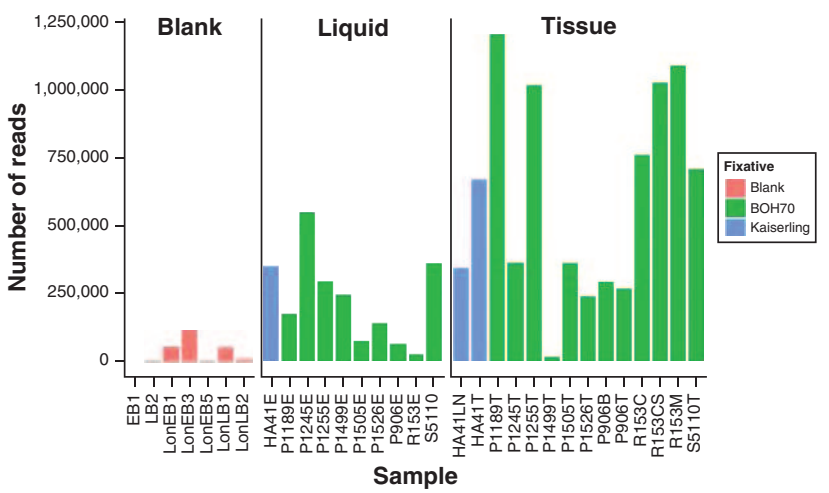

(D) Source-sink community composition

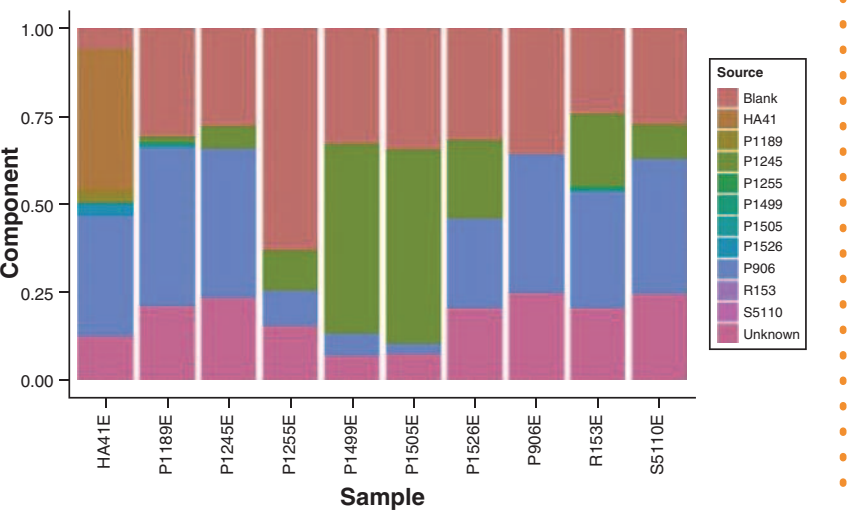

Figure 2. (A) Damage pattern of the mtDNA reads from sample HA4.1 after hybridization capture. (B) Total number of bacterial reads on each sample. (C) Main bacterial phyla present in the samples, expressed in abundance. (D) Bacterial community composition expressed in percentage component by SourceTracker2.

of the families present in the tissue (Enterobacteriaceae, 22\%, and Methylobacteriaceae, 12\%) (Supplementary Figure 2). HA4.1 was further characterized by being the only fixative sample with similar bacteria as in the tissue samples, such as Klebsiella. When the authors applied SourceTracker2 [18] to identify the proportion of the liquid bacteria community explained by the tissue samples, HA4.1 had a higher presence of taxa from the tissues, contributing $40 \%$ of the bacteria present in the HA4.1 fixative. It is noteworthy that the main contributors in the liquid community were the non-template controls, contributing from $30 \%-70 \%$ of the bacterial community in the liquid. This shows the strong impact of trace bacterial DNA even from sterile reagents in low-mass DNA studies (Figure 2D), including the unknown metagenomic content of the fixative material alone. Future assessment of virgin fixative material could be of use for estimating the contribution of environmental taxa.

To test the viability of detecting pathogen DNA, the authors performed pathogen-specific hybridization captures for $M$. tuberculosis, M. leprae and T. pallidum subsp. pallidum (Supplementary Note 2). Only the M. tuberculosis and M. leprae enrichments were successful, while the T. pallidum protocol did not yield any usable output (Supplementary Tables 9 \& 10). For the fixative material from HA4.1, only 790 reads were detected, with a damage pattern of $22 \%$ ( $0.082 \%$ endogenous DNA, 0.3 coverage), which did not provide enough coverage for a more in-depth analysis of the pathogen. Despite having the damage profile characteristic of ancient reads, with a cluster factor of 10.29 at $5 \%$ of a lane, the authors considered that deeper sequencing would not provide better results to discern whether the reads were truly endogenous ancient $M$. leprae or corresponded to environmental contamination presenting damage from reasons other than age [19]. Given that the mtDNA enrichment was successful for the tissues and one liquid sample, the authors believe that these tissue samples were not good candidates for the characterization of bacterial aDNA. Therefore, the testing of pathogen DNA leaching was not successful with this sample set.

Several limitations arise from this study: the success rate was low, with only one sample (HA4.1) presenting retrievable human reads from the fixative material, and that had a bacterial profile that was $40 \%$ similar to the one characterized in the HA4.1 tissue sample. As a small-scale study with a limited sample size, this approach proved to be successful for extracting host aDNA, but the recovery of bacterial DNA, either commensal or pathogenic, was more complicated to achieve. Some potential solutions to improve the technique, 
such as increasing the fixative volume extracted, could be implemented for future studies. As an initial screening, the authors extracted between 1 and $2 \mathrm{ml}$ of liquid fixative, and they believe that a larger starting amount could increase the amount of DNA in suspension. Furthermore, factors such as the proportion of the tissue surface to total fixative volume could play a role in the amount of DNA released over time, since larger tissues in smaller volumes would present a relatively larger exposed surface. Such examinations have not been included here, but future studies would benefit greatly from large-scale experimental tests of these factors. In addition, despite the amount of detail about the history of the sample that archives can provide, the maintenance and exchange of the fixative material were typically not recorded. Since each exchange could remove suspended DNA, samples with 'older' fixative liquid would theoretically contain more leached DNA. Also, it seems that the type of fixative could impact the transfer of DNA. When explaining this apparent success, the authors revisited the information detailing the fixative conditions (Supplementary Table 1). The fact that this sample was stored in Kaiserling solution instead of $70 \%$ ethanol may have contributed to the preferential preservation of free DNA from the host in the liquid content. This fixative is generally an aqueous solution of formalin, potassium nitrate and potassium acetate, although several variations were commonly found among collections (Supplementary Note 4). However, as is common with historical archival sources, the history of these samples is somewhat incomplete and it is possible that other liquid fixatives were added throughout the centuries (Supplementary Note 4). Alternatively, it could be a simple matter of better preservation of this specific sample, independent from the type of fixative. Further selection and analysis of more samples preserved in Kaiserling solution would elucidate if this fixative procedure is a consistent and reproducible source for leached DNA.

In conclusion, here the authors present the potential use of liquid fixative materials from historical collections as a source for ancient DNA studies. Although this approach could become a non-destructive surveying method in the future, these results highlight the need to study the nature of the fixative liquids in more detail and develop criteria under which the tissue itself should be sampled after DNA retrieval from the fixative liquid is not successful. The authors' recommendations for future studies are to select specimens whose fixative material had been changed less frequently, estimated either through the recorded history of the specimen or visually (more turbid samples); to sample larger volumes of fixative material (circa $5 \mathrm{ml}$ ) to maximize the recovery of DNA in suspension; if possible, to select specimens that proportionally have a larger surface exposed to the liquid; and to test different types of fixative material to test differential success on DNA preservation. Overall, the authors demonstrated that it is possible to retrieve host aDNA from preserved samples without destruction of the embedded tissue, opening a new array of possibilities for the molecular analysis of medical and historical collections.

\section{Supplementary data}

To view the supplementary data that accompany this paper please visit the journal website at: www.future-science.com/doi/suppl/10. 2144/btn-2021-0014

\section{Author contributions}

E Rayo and G Ferrari conceived the presented idea. M Cooke and C Phillips provided samples and historical context. E Rayo, G Ferrari, AM Breidenstein and G Akgül carried out the laboratory work. E Rayo and G Ferrari wrote the manuscript with input from VJ Schuenemann, AM Breidenstein and M Cooke. J Neukamm and E Rayo performed the metagenomic analysis and data intepretation. G Ferrari, E Rayo and AS Bouwman obtained the funding for the project. VJ Schuenemann, FJ Rühli and AS Bouwman supervised the project. All authors provided critical feedback and helped shape the research, analysis and manuscript.

\section{Acknowledgments}

The authors would like to thank the Swiss Mäxi-Foundation for its support. They also thank the Functional Genomics Center of Zürich (FGCZ) for the sequencing service provided.

\section{Financial \& competing interests disclosure}

This project was funded by the University of Zürich, Switzerland, as a pilot project under the University Research Priority Program: Evolution in Action funding scheme. The authors have no other relevant affiliations or financial involvement with any organization or entity with a financial interest in or financial conflict with the subject matter or materials discussed in the manuscript apart from those disclosed.

No writing assistance was utilized in the production of this manuscript.

\section{Open access}

This work is licensed under the Attribution-NonCommercial-NoDerivatives 4.0 Unported License. To view a copy of this license, visit http://creativecommons.org/licenses/by-nc-nd/4.0/

\section{References}

1. Cobb JC. Ancient DNA recovered by a non-destructive method. Anc. Biomol. 4(4), 169-172 (2002).

2. Thomsen PF, Elias S, Gilbert MTP et al. Non-destructive sampling of ancient insect DNA. PLoS One 4(4), e5048 (2009). 
3. Rohland N, Siedel H, Hofreiter M. Nondestructive DNA extraction method for mitochondrial DNA analyses of museum specimens. BioTechniques 36(5), 814-816, 818-821 (2004).

4. Rohland N, Hofreiter M. Ancient DNA extraction from bones and teeth. Nat. Protoc. 2(7), 1756-1762 (2007).

5. Ficetola GF, Miaud C, Pompanon F, Taberlet P. Species detection using environmental DNA from water samples. Biol. Lett. 4(4), 423-425 (2008).

6. Taberlet P, Coissac E, Hajibabaei M, Rieseberg LH. Environmental DNA. Mol. Ecol. 21(8), 1789-1793 (2012).

7. Shokralla S, Singer GAC, Hajibabaei M. Direct PCR amplification and sequencing of specimens' DNA from preservative ethanol. BioTechniques 48(3), 233-234 (2010).

8. Neukamm J, Peltzer A, Nieselt K. DamageProfiler: fast damage pattern calculation for ancient DNA. Bioinformatics (2021). http://dx.doi.org/10.1093/bioinformatics/btab190

9. Maricic T, Whitten M, Pääbo S. Multiplexed DNA sequence capture of mitochondrial genomes using PCR products. PLoS One 5(11), e14004 (2010).

10. Renaud G, Slon V, Duggan AT, Kelso J. Schmutzi: estimation of contamination and endogenous mitochondrial consensus calling for ancient DNA. Genome Biol. 16, 224 (2015).

11. Blair TL. The shaping of Black London to the 21st Century. Editions Blair. ISBN 978-1-908480-16-3, The Black London eMonograph Series (2013).

12. Weissensteiner H, Pacher D, Kloss-Brandstätter A et al. HaploGrep 2: mitochondrial haplogroup classification in the era of high-throughput sequencing. Nucleic Acids Res. 44(W1), W58W63 (2016).

13. McMurdie PJ, Holmes S. phyloseq: an R package for reproducible interactive analysis and graphics of microbiome census data. PLoS One 8(4), e61217 (2013).

14. Cousin MA. PSEUDOMONAS | Introduction. In: Encyclopedia of Food Microbiology. Robinson RK (Ed.). Elsevier, Oxford, UK, 1864-1867 (1999).

15. Octavia S, Lan R. The family Enterobacteriaceae. In: The Prokaryotes: Gammaproteobacteria. Rosenberg E, DeLong EF, Lory S, Stackebrandt E, Thompson F (Eds). Springer Berlin Heidelberg, Berlin, Heidelberg, Germany, 225-286 (2014).

16. Voronina OL, Kunda MS, Ryzhova NN et al. The variability of the order Burkholderiales representatives in the healthcare units. Biomed Res. Int. 2015, 680210 (2015).

17. Figueiredo GGO, Lopes VR, Romano T, Camara MC. Chapter 22 - Clostridium. In: Beneficial Microbes in Agro-Ecology. Amaresan N, Senthil Kumar M, Annapurna K, Kumar K, Sankaranarayanan A (Eds). Academic Press, MA, USA, 477-491(2020).

18. Knights D, Kuczynski J, Charlson ES et al. Bayesian community-wide culture-independent microbial source tracking. Nat. Methods 8(9), 761-763 (2011).

19. Bouwman AS, Kennedy SL, Müller R et al. Genotype of a historic strain of Mycobacterium tuberculosis. Proc. Natl Acad. Sci. USA 109(45), 18511-18516 (2012). 